\title{
Ultrasound study of heel to calcaneum depth in neonates
}

Anoo Jain, Nicholas Rutter

\begin{abstract}
Aim-To investigate whether it would be safe to extend the currently recommended area of sampling to the whole heel in neonates.

Methods-Eighty newborn infants were studied, weight range 0.56 to $4.34 \mathrm{~kg}$, gestation 24 to 42 weeks. Ultrasound scanning was used to measure the shortest distance between the skin and the perichondrium of the calcaneum.

Results-The shortest depth of perichondrium was in the centre of the heel and ranged from 3 to $8 \mathrm{~mm}$. In 78 of the 80 infants the distance was $4 \mathrm{~mm}$ or more. There was a small but significant positive correlation with weight.

Conclusions-Standard automated lancets for preterm use that puncture to a depth of $2.4 \mathrm{~mm}$ may be safely used anywhere over the plantar surface of the heel. The posterior aspect of the heel should be avoided. Reducing the density of heel pricks should reduce the associated pain.
\end{abstract}

(Arch Dis Child Fetal Neonatal Ed 1999;80:F243-F245)

Keywords: heel prick; calcaneum; site of puncture; pain

Blood sampling is important in the management of newborn infants. All infants undergo biochemical screening; many healthy term and preterm infants will need monitoring of bilirubin and glucose, and infants in intensive care may need over 100 blood tests as part of their management.

Peripheral venepuncture is a skilled task which takes time to master and needs ongoing practice. Capillary heel prick blood sampling is quick, easy to learn, and to perform. It is therefore by far the most common method of blood sampling in neonates. Unfortunately, it is also

Department of

Neonatal Medicine, Nottingham City

Hospital,

Nottingham NG5 1PB

A Jain

Division of Child

Health,

School of Human

Development,

Queen's Medical

Centre,

Nottingham

N Rutter

Correspondence to:

Dr Anoo Jain.

Email:

anoo.jain@nottingham.ac.uk

Accepted 29 October 1998 Echodense area is ossified bone. painful, probably more painful than venepuncture if manual lances are used. ${ }^{2}$ Reduction in the pain caused by this technique is important. Attention has focused on the use of different devices for heel pricking as a means of reducing pain, ${ }^{3-5}$ but the site of sampling has been ignored.

Current guidelines for the site of heel prick blood sampling are based on a study by Blumenfeld in $1979 .{ }^{6}$ These recommend that samples are taken from a limited area on the lateral borders of the plantar surface of each heel to avoid puncture (and thus infection) of the calcaneum. This means that heel pricks are concentrated together, particularly in low birthweight infants with small feet. Tissue injury caused by the lance results in inflammatory change which makes the area tender and therefore hypersensitive to further pricks.

Blumenfeld's guidelines are based on post mortem measurement of heel to calcaneum depth and microscopic examination of heel tissue. Since they were published, there has been an increase in the number of very premature infants and consequently a large increase in the number of heel pricks. ${ }^{1}$ In many units manual blade lances have been replaced with spring loaded lances which penetrate to a fixed depth. In spite of these changes, the original guidelines have never been reviewed. We therefore conducted a study in live infants to determine whether it would be safe to use any part of the plantar surface of the heel for blood sampling.

\section{Methods}

An Ultramark 4A ultrasound machine (ATL Corp., USA) was used with a $7.5 \mathrm{Mhz}$ scan head. A spacing device was placed securely over the plantar surface of the heel and the calcaneum was imaged through this. A pilot study in 10 infants showed that the interfaces between skin surface, perichondrium, and
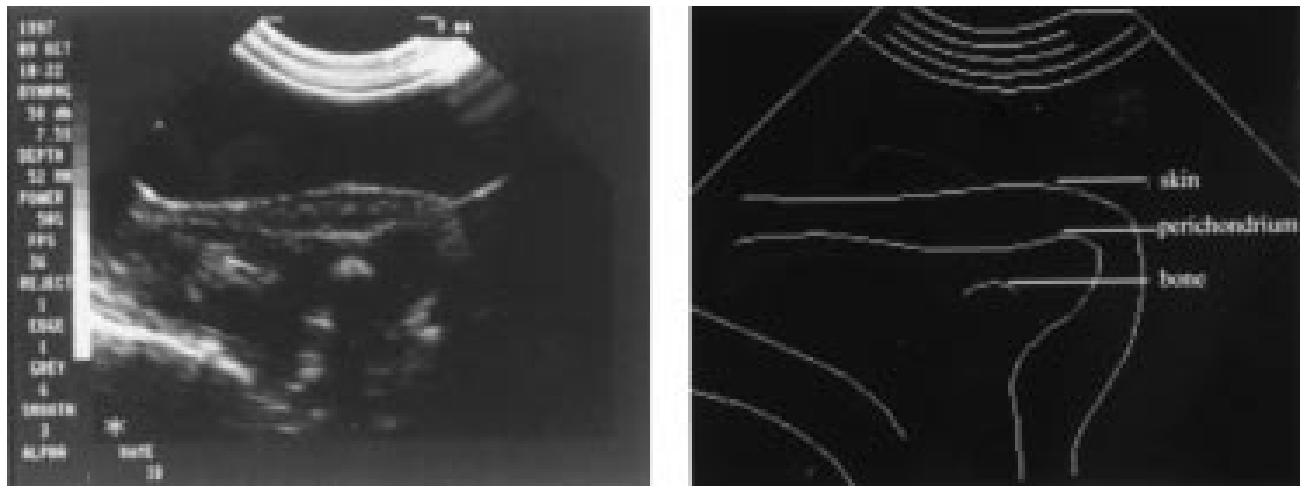

Figure 1 Ultrasound view of heel in sagittal axis. Translucent area under skin is cartilaginous part of calcaneum. 


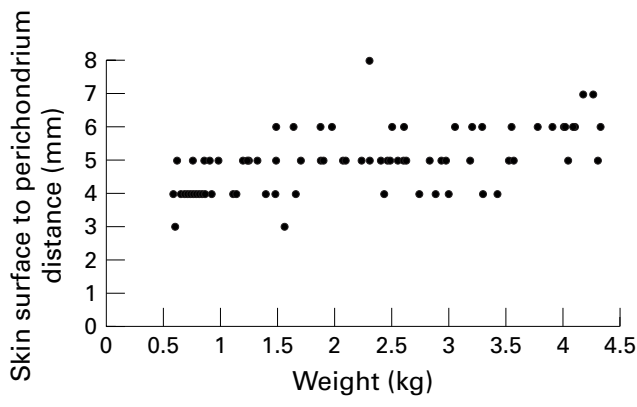

Figure 2 Shortest distance from skin surface to perichondrium plotted against weight. Standard spring loaded lancet used in preterm infants penetrates to a depth of $2.4 \mathrm{~mm}$.

osseous calcaneum could be clearly defined (fig 1). The calcaneum was rounded in shape, with its convex surface facing the plantar surface of the heel. There was a single point at which the calcaneum was closest to the skin surface. To define this point, the shortest distance from the skin to perichondrium was recorded in the coronal and saggital planes. The electronic callipers of the ultrasound machine were used to measure this distance. All measurements were performed by the same observer. The coefficient of variation for repeated measurements on the same infant was $8 \%$.

Eighty newborn infants were studied. To achieve a full range of size we stratified subjects by birthweight into four groups of 20: less than $1000 \mathrm{~g}, 1001-2000 \mathrm{~g}, 2001-3000 \mathrm{~g}$ and greater than $3001 \mathrm{~g}$. The range of weight was $0.56 \mathrm{~kg}$ to $4.34 \mathrm{~kg}$, gestation 24 to 42 weeks. Measurements were made in the first week after birth.

\section{Results}

The shortest skin to perichondrium distance ranged from 3 to $8 \mathrm{~mm}$ (fig 2). In 78 of the 80 infants it exceeded $4 \mathrm{~mm}$. In one infant of 0.61 $\mathrm{kg}$ and one of $1.56 \mathrm{~kg}$ the distance was $3 \mathrm{~mm}$. Linear regression analysis showed a line of best fit with a gradient of 0.45 , significantly different from the horizontal $(p<0.0001)$. There was a significant but small positive influence of birthweight. The cartilaginous content of the calcaneum was high in all infants. The
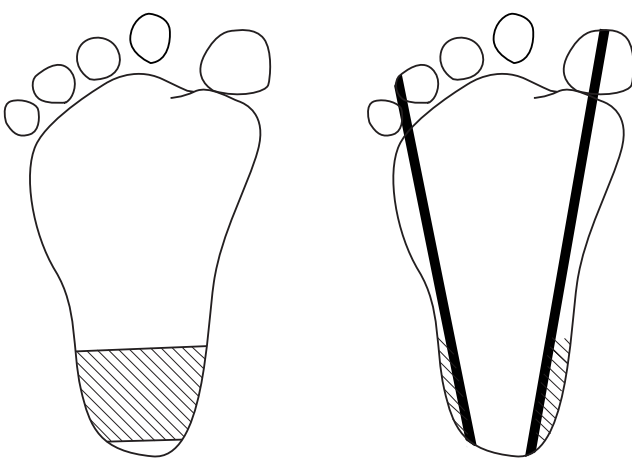

Figure 3 Current recommendations for site of heel puncture. Side to side limits of calcaneum are marked by a line extending posteriorly from a point between 4 th and 5th toes running parallel to lateral aspect of heel and a line extending posteriorly from middle of big toe running parallel to medial aspect of the heel. Shaded area shows recommended site of puncture. ${ }^{6}$ degree of ossification increased with weight and gestational age - in the most immature infants it was barely present.

\section{Discussion}

The calcaneum has been studied by ultrasound scanning live newborn infants over a wide weight range. Our technique was reproducible and readily delineated the skin, perichondrium, and osseous calcaneum. We found that the calcaneum is rounded in shape, with its convexity facing the plantar heel surface. Posteriorly it is wider and much closer to the surface of the skin. We measured the point at which the perichondrium was closest to the plantar aspect of the heel because this is the site where calcaneal puncture would be most likely to occur. This distance was at least $3 \mathrm{~mm}$ in all the infants studied, and usually considerably more even in the smallest infants. Because of the curved shape of the calcaneum, this shortest distance is only found at one point on the plantar surface of heel. At all other points the distance from skin to perichondrium is greater. We were surprised to find that the shortest distance between skin and perichondrium was not strongly influenced by weight. The soft tissue of the heel is well developed in the smallest infants. The degree of ossification of the calcaneum varied with birthweight - in the smallest babies it was barely present but in the larger ones it was well formed. We assume that this is a marker of maturity.

A recent study showed that heel prick blood sampling was the commonest invasive procedure performed in neonatal intensive care. ${ }^{1}$ Heel pricks (3283) were performed on 54 infants. One infant born at 23 weeks of gestation had a total of 218 heel pricks. Our experience is that some infants on postnatal wards might have up to 20 heel pricks for glucose and bilirubin measurement while in hospital.

Recommendations on the site of heel prick sampling were published by Blumenfeld in $1979^{6}$ to reduce the incidence of osteomyelitis of the calcaneum secondary to heel prick blood sampling. This postmortem study examined the heels of 40 children weighing between 0.56 and $13.15 \mathrm{~kg}$. A vernier calliper was used to measure the skin to perichondrium distance from histological specimens. The minimum skin to perichondrium distance was $2.4 \mathrm{~mm}$ on the plantar surface and half this over the posterior aspect of the heel. The vascular bed of the skin lay 0.35 to $1.6 \mathrm{~mm}$ below the skin surface. There was one case of focal necrotising chondritis in a baby who already had an abscess of the heel before heel prick sampling. In other cases the perichondrium had been injured without causing infection. These findings led to the publication of guidelines on blood sampling from the heel which are still in use (fig 3).

Although osteomyelitis of the calcaneum is a serious complication of heel prick blood sampling, the number of cases reported is very small and none is recent. Lauer reported five cases of staphylococcal osteomyelitis that were thought to have resulted from heel prick blood 
sampling. ${ }^{8}$ Lancet lengths were 2.5 to $4.9 \mathrm{~mm}$ and the posterior area of the heel was used if numerous punctures had already been performed (there is no documentation of the length or type of lancet used for each baby). Defects in the initial skin cleansing, use of the posterior curve of the heel, and inadequate technical quality control were thought to have played a part in the development of the infection. When these were corrected, no further cases of calcaneal osteomyelitis were reported from that institution. Two cases of staphylococcal calcaneal osteomyelitis resulting from multiple capillary heel prick blood sampling were reported by Lilien et al. ${ }^{9}$ Reduction of growth of the hind foot resulting from calcaneal osteomyelitis was reported by Borris. ${ }^{10}$

Blumenfeld's study was valuable in providing guidelines for sampling from the heel, but it has led to the crowding of multiple punctures into a small area along the lateral borders of each heel. Now that very small infants are receiving prolonged intensive care, their heels are becoming sore because the multiple punctures are confined to such a small area of the heel borders. There is evidence that this leads to hypersensitivity with the result that each subsequent heel prick becomes more painful. ${ }^{7}$ The use of automated, spring loaded lancets or blades in the newborn period has increased. These devices provide a rapid puncture or incision to a predetermined fixed depth and are suitable for routine use in term and preterm infants. Their use reduces the pain of heel pricks when compared with manual lances. ${ }^{3-5}$ The standard spring loaded lance in common use in preterm infants (Autolet, Owen Mumford, UK) penetrates to a controlled depth of $2.4 \mathrm{~mm}$. Our work suggests that even in the smallest infants, it would be safe to use this device (and any other with a similar depth of penetration) over the whole of the plantar surface of the heel. The posterior surface should be avoided because the calcaneum is too superficial at this site. If the whole of the plantar surface of the heel is used, the pricks can be spaced out and recently traumatised areas avoided. This should reduce some of the pain associated with heel prick sampling.

1 Barker DP, Rutter N. Exposure to invasive procedures in neonatal intensive care unit admissions. Arch Dis Child 1995:72:F47-8

2 Shah VS, Taddio A, Bennett S, Speidel BD. Neonatal pain response to heel stick vs venepuncture for routine blood
sampling Arch Dis Child 1997;77:F143-4.

3 Harpin VA, Rutter N. Making heel pricks less painful Arch Dis Child 1983; 58:226-8.

4 McIntosh N, van Veen L, Brameyer H. Alleviation of the pain of heel prick in preterm infants. Arch Dis Child pain of heel prick

5 Barker DP, Latty BW, Rutter N. Heel blood sampling in preterm infants: which technique? Arch Dis Child 1994:71:F206-8

6 Blumenfeld TA, Turi GK, Blanc WA. Recommended site and depth of newborn heel skin punctures based on
anatomical measurements and histopathology. Lancet 1979;i:230-3.

7 Fitzgerald M, Millard C, McIntosh N. Cutaneous hypersensitivity following peripheral tissue damage in newborn infants and its reversal with topical anaesthesia. Pain 1989;39:31-6.

8 Lauer BA, Altenburger KM. Outbreak of staphylococcal infections following heel puncture for blood sampling. Am f Dis Child 1981:135:277-8.

9 Lilien LD, Harris VJ, Ramamurthy RS, Pildes RS. Neonatal osteomyelitis of the calcaneus: complication of heel puncture. F Pediatr 1976;88:478-80.

10 Borris LC, Helleland H. Growth disturbance of the hind part of the foot following osteomyelitis of the calcaneus in part of the foot following osteomyelitis of the calcaneus in 1986:68:302-5. 